\title{
Factores Asociados a Hemorragia Intraventricular En Neonatos Atendidos En Un Hospital de Segundo Nivel
}

\section{Miguel Ángel Martínez Camacho Alberto Juárez Lira}

Especialidad en Salud Pública de la Universidad Autónoma de Querétaro, México

Ma. de los Ángeles López Cortéz

Hospital de Especialidades del Niño y la Mujer-SESEQ, México

\section{Helios Mancera Roque}

Especialidad en Salud Pública de la Universidad Autónoma de Querétaro, México

Hospital de Especialidades del Niño y la Mujer-SESEQ, México

Doi: 10.19044/esj.2017.v13n36p71 URL:http://dx.doi.org/10.19044/esj.2017.v13n36p71

\begin{abstract}
Intraventricular hemorrhage (HIV) is a complication in neonates. It originates in the subependymal germinal matrix. This area is irrigated by a network of poorly differentiated vessels without a basement membrane. Also, it is fragile and vulnerable to the hemodynamic instability of cerebral blood flow. It is an important risk factor for periventricular leukomalacia, ventriculomegaly, and hydrocephalus. These neurological pathologies can generate sequelae in the pediatric population such as cerebral palsy (CP). Objective: To identify the factors associated with HIV in neonates treated in the early intervention program of the hospital of specialties of the child and the woman of the Secretary of Health of the State of Querétaro (HENMSESEQ). Material and Methods: Through the review of the clinical files, the factors associated with HIV present in the children attended to in the HENMSESEQ early intervention service were observed in 2015. Cases of HIV were corroborated by means of transfontanel ultrasound, statistical analysis with measures of central tendency, and comparison of the medians of children diagnosed with HIV and without diagnosis. Results: A group of 69 infants attended to in the early intervention program was studied. Out of them, $65.2 \%$ (45) were women and $34.3 \%$ (24) were men. They had a gestational age of $34.19 \pm 4.22$ weeks, birth weight of $2116.09 \pm 859.36 \mathrm{~g}$, and height of $44.06 \pm 6.02 \mathrm{~cm}$. Their stay in the Neonatal Intensive Care Unit (NICU) for the sample was $11.88 \pm 16.84$ days and they were $8.65 \pm 12.20$ days with invasive mechanical ventilation. There were 25 cases of HIV diagnosed by
\end{abstract}


transfontanel ultrasound. Out of the 25 cases, 7 (10\%) were HIV grade I, 14 (20\%) II, $4(5.7 \%)$ III, and no cases of grade IV were diagnosed. Using the Mann-Whitney $U$ test, a difference was found between the groups of neonates diagnosed with HIV and without diagnosis in weeks of gestation, height, weight, APGAR, days of intubation, and days of stay in NICU.

Keywords: Intraventricular hemorrhage, newborn, gestational age, neonatal intensive care unit

\section{Resumen}

La hemorragia intraventricular (HIV) es una complicación en los neonatos, se origina en la matriz germinal subependimaria. Esta zona está irrigada por una red de vasos poco diferenciados, sin membrana basal, frágil y vulnerable a la inestabilidad hemodinámica del flujo sanguíneo cerebral. Es un factor de riesgo importante para leucomalacia periventricular, ventrículomegalia e hidrocefalia. Estas patologías neurológicas pueden generar secuelas en la población pediátrica como es la parálisis cerebral (PC). Objetivo. Identificar los factores asociados a HIV en neonatos atendidos en el programa de intervención temprana del hospital de especialidades del niño y la mujer de la secretaria de salud del estado de Querétaro (HENM-SESEQ). Material y Métodos. Por medio de la revisión de los expedientes clínicos se observaron los factores asociados con HIV presentes en los niños atendidos en el servicio de intervención temprana del HENM-SESEQ en al año 2015. Se corroboraron casos de HIV por medio de ultrasonido transfontanelar, posteriormente se realizó análisis estadístico con medidas de tendencia central y comparación de medianas de los niños con diagnóstico de HIV y sin diagnóstico. Resultados. Se estudió un grupo de 69 neonatos atendidos en el programa de intervención temprana. De los cuales el $65.2 \%$ (45) son mujeres y el $34.3 \%$ (24) son varones. Tuvieron una edad gestacional de $34.19 \pm 4.22$ semanas, peso al nacer de $2116.09 \pm 859.36 \mathrm{~g}$ y talla de $44.06 \pm 6.02 \mathrm{~cm}$. La estancia en la Unidad de Cuidados Intensivos Neonatales (UCIN) para la muestra fue de $11.88 \pm 16.84$ días y estuvieron $8.65 \pm 12.20$ días con ventilación mecánica invasiva. Se observaron 25 casos de HIV diagnosticados por medio de ultrasonido transfontanelar de los cuales $7(10 \%)$ son HIV grado I, 14 (20\%) II, 4 (5.7\%) III y no se diagnosticaron casos de grado IV. Utilizando la U de Mann-Whitney se encontró diferencia entre los grupos de neonatos con diagnóstico de HIV y sin diagnóstico en: semanas de gestación, talla, peso, APGAR, días de intubación y días de estancia en UCIN.

Palabras claves: Hemorragia intraventricular, recién nacido, edad gestacional, unidad de cuidados intensivos neonatales 


\section{Introducción}

Durante la década de los 90`s, la implementación de nuevas intervenciones terapéuticas perinatales y neonatales mejoró de manera notable la supervivencia de los neonatos prematuros, en particular la del grupo de extremo bajo peso al nacimiento $(<1000 \mathrm{~g})$ (Basso, 2016). Este grupo de pacientes presentan las mayores tasas de comorbilidad global y neurológica y, en consecuencia, es objeto de mayor preocupación por parte de obstetras, neonatólogos, las familias y la sociedad en conjunto (Euson, 2012). Por otra parte las complicaciones respiratorias como el síndrome de distres respiratorio y displasia broncopulmonar contribuyen a un peor pronóstico y hospitalización más prolongada en la UCIN (Christian et al., 2016).

La HIV es una complicación común en el recién nacido pretermino (RNPT). Los neonatos prematuros son especialmente frágiles a la aparición de HIV debido a la vulnerabilidad de la matriz germinal. (Wusthoff, 2016) Los estudios neuroevolutivos en estos niños han mostrado que los grados más graves de HIV y/o leucomalacia periventricular (LPV) son los mejores predictores de evolución neurológica anormal. El daño cerebral del prematuro y sus consecuencias resultan de un intricado proceso que incluye destrucción neuronal, detenimiento del desarrollo neurológico, reparación y reorganización aberrante de la conectividad cerebral (Basso, 2016).

Las complicaciones de la HIV pueden ocasionar alteraciones en el neurodesarrollo, como son el infarto hemorrágico periventricular (IHP) y ventriculomegalia poshemorrágica (VPH). Con frecuencia, la HIV se asocia con lesión de las sustancia blanca (LSB) y LPV (Ahn \& Shim, 2016). El pronóstico de los pacientes con HIV depende en gran medida de la severidad de la hemorragia. Históricamente las hemorragias severas están asociadas con más del $20 \%$ de la mortalidad y el riesgo mayor de alteración en el neurodesarrollo incluyendo PC, ceguera y retraso motor o cognitivo (Wusthoff, 2016).

La incidencia de la HIV, a pesar de haber ido disminuyendo está en torno al 20-25\% para el grupo de RNPT < 1500 g. Sin embargo, la relevancia del problema no ha disminuido por dos razones fundamentales: 1) la incidencia se relaciona directamente con el grado de prematuridad; es del $45 \%$ en aquellos entre 500 y $750 \mathrm{~g} \mathrm{y}, 2$ ) al aumento de la tasa de supervivencia que es del $70 \%$ en aquellos con peso entre 500 y $1000 \mathrm{~g}$ (Basso, 2016). La HIV severa ocurre casi de manera exclusiva a infantes menores de 28 semanas de gestación. La incidencia es del $27 \%$ en neonatos menores de 26 semanas y menos del $2 \%$ en niños mayores de 28 semanas (Liebowitz \& Clyman, 2016). Los pacientes con menos de 22 SDG tienen una incidencia de HIV del 70\%. La edad gestacional se observa que tiene una significancia mayor que el peso al nacer. La incidencia, así como la 
severidad de la HIV, disminuye con el aumento de la edad gestacional y el peso al nacer.(Ahn et al., 2015)

La HIV es multifactorial ya que depende de alteraciones de origen materno, intraparto y condiciones propias del recién nacido. (Cervantes et al., 2012). El 56\% de los pacientes con HIV en un estudio americano son varones. El $41 \%$ de los neonatos prematuros con HIV tuvieron un peso al nacer entre 500 a $999 \mathrm{~g}$. El grupo racial con mayor número de casos fueron los blancos (34\%), seguido de los niños negros (19\%) y los hispanos (15\%) (Christian et al., 2016).

En los neonatos pretérmino, los vasos sanguíneos de la matriz germinal tienen una estructura poco resistente, lo cual incrementa la posibilidad de ruptura, especialmente con alteraciones en la regulación de la circulación cerebral (Cervantes et al., 2012). La hemorragia desde el plexo coroideo no es tan habitual, y ocurre sobre todo en los RNPT más maduros (Wusthoff, 2016).

Los estudios neuropatológicos sugieren que la hemorragia en la matriz germinal se produce primero en la red capilar, que comunica libremente con el sistema venoso, aunque el sangrado también puede ocurrir desde la vasculatura arterial. Los vasos en esta región ocupan zonas limítrofes entre las arterias cerebrales y el área de drenaje de las venas cerebrales profundas. Además, la permeabilidad aumenta en situaciones de hipoxia o ante el incremento de la presión venosa (Christian et al., 2016).

De manera clásica se ha utilizado la escala de Papile para describir la severidad de la HIV (Ferriero, 2016). Grado I: hemorragia limitada en el surco caudotalámico. Grado II: se extiende a la luz ventricular, sin dilatar su tamaño. Grado III: presenta dilatación ventricular desde el inicio. Grado IV: existe afectación parenquimatosa asociada a infarto venoso (Moreno, 2016). Sin embargo esta clasificación no toma en cuenta la severidad del daño del parénquima (excepto el grado IV), tampoco toma en cuenta si la hemorragia es bilateral, que sin lugar a dudas tiene un impacto en el pronóstico del neurodesarrollo del paciente. Se requiere una forma de evaluación que describa de manera más precisa la severidad del daño (Wusthoff, 2016).

Existen diversos factores de riesgo asociados a HIV como la corioamnionitis, hipotensión arterial o fluctuaciones en la misma, síndrome de distres respiratorio con hipocapnia, hipercapnia o hipoxemia, ventilación mecánica y terapia con bicarbonato. Además de bajo peso al nacer, prematurez y ser varón (Glauco, 2015). El 6\% de los pacientes con HIV presentaron convulsiones durante la admisión inicial, además se reportó que un $38 \%$ de los niños estudiados contaban con algún defecto cardiaco congénito. Se observó que la población tenía las siguientes comorbilidades: $8 \%$ enterocolitis necrosante, $4 \%$ perforación intestinal, $85 \%$ complicaciones pulmonares secundaria que requirieron de ventilación mecánica o soporte 
respiratorio adicional, $68 \%$ complicaciones hematológicas como la coagulación intravascular diseminada. Mientras que la HIV es la alteración patológica más frecuente, la lesión de la LSB hemisférica es la característica cardinal del daño cerebral en el RNPT, al ser esta la principal alteración que se objetiva, tanto en el período neonatal como en etapas posteriores y que parece responsable de los déficits motores espásticos, cognitivos, del aprendizaje y neurosensoriales mayores que se observan en los supervivientes (Basso, 2016).

En un estudio coreano la incidencia de HIV en neonatos con muy bajo peso al nacer fue del 42.2\%. Según los grados I $25.1 \%$, II 7\%, III 4.8\% y IV 5.5\%. Los niños con hemorragia intraventricular grave (grado III y IV) tienen un peor pronóstico en cuanto a neurodesarrollo que los niños con hemorragia grado I y II o sin presencia de dicho diagnóstico. A mayor severidad, según la escala de Papile, mayor impacto en el neurodesarrollo o muerte (Ahn et al., 2015). Alrededor del 15\% de las HIV asociarán lesiones intraparenquimatosas dorsalmente y lateralmente al ángulo externo del ventrículo lateral. Su incidencia aumenta con la disminución de la edad gestacional, aparece en el $80-90 \%$ de las HIV extensas, suelen ser asimétricas (unilaterales), y el 50\% son extensos al ocupar la sustancia blanca periventricular desde frontal a perietooccipital. El resultado ultrasonográfico sugestivo es la presencia de un área ecodensa de forma globular o en abanico, en general es unilateral o, si es bilateral, es claramente asimétrica, en la sustancia blanca adyacente a los ventrículos laterales (Moreno, 2016).

La muerte es la mayor complicación de la HIV, estudios recientes reportaran una mortalidad aproximada del $30 \%$ en neonatos con HIV grado III y del 60\% con HIV grado IV (Ahn et al., 2015). Los factores que contribuyen a predecir muerte y alteraciones del neurodesarrollo incluyen la severidad, la localización (bilateral vs unilateral) y presencia de infarto en parénquima derivado de la hemorragia (Mukerji, Shah \& Shah, 2015). El riesgo de PC está claramente asociado con el grado de HIV (50\% en HIV IV vs $8 \%$ en HIV I) (Wusthofff, 2016). El IHP es la complicación más grave de la hemorragia intraventricular y resulta en grave discapacidad (Ferriero, 2016). La relación patogénica del IHP con la HIV se base en: $80-90 \%$ de las lesiones en parénquima se asocian a HIV extensas y asimétricas, las lesiones homolaterales donde está la HIV extensa y, en algunos casos, las lesiones en parénquima aparecen y progresan al producirse una HIV. Estos datos sugieren que la HIV lleva a la obstrucción de las venas terminales y, por ello, a la interrupción del flujo sanguíneo en las venas medulares con el consiguiente infarto hemorrágico venoso, que suele producirse con rapidez (Basso, 2016). 
Por todo lo anterior es necesario reconocer los principales factores asociados a HIV en la población atendida en el programa de intervención temprana del HENM-SESEQ debido a la vulnerabilidad neurológica y al riesgo de padecer una discapacidad permanente que presenta el RNPT.

\section{Material y Método}

\section{Diseño del Estudio}

Estudio observacional, transversal, descriptivo y retrospectivo. El marco muestral se obtuvo de los expedientes clínicos de los pacientes atendidos en el año 2015 en el servicio de intervención temprana del HENMSESEQ, México.

\section{Tamaño de la Muestra}

La población estudiada estuvo formada por los niños con expediente completo y que contaran con ultrasonido transfontanelar. Fueron eliminados del estudio los pacientes que no nacieron en dicho hospital o que tuviesen el expediente incompleto, quedando un total de 69 niños, de los cuales 25 tenían HIV.

\section{Procedimiento}

El protocolo se sometió a evaluación por parte del comité de investigación del Hospital de Especialidades del Niño y la Mujer perteneciente a la Secretaria de Salud del Estado de Querétaro, México obteniendo la autorización para la realización del mismo.

Se revisaron los expedientes clínicos del 2015 de manera individual buscando las variables de interés: peso al nacer, semanas de gestación, talla, días de estancia en la UCIN, además de diagnóstico de HIV corroborado por ultrasonido transfontanelar. Debido a las características del estudio se verificaron las notas de evolución, hoja de enfermería y estudios de laboratorio, radiológicos y de gabinete. Posteriormente la información se acomodó en tablas de Excel para su posterior análisis en IBM SPSS Statistics 21.

\section{Análisis de los Datos}

Se realizó medidas de tendencia central, además de tablas de frecuencias y porcentajes de las variables de interés en la población general y en los niños con diagnóstico de HIV. Posteriormente se hizo prueba de normalidad en las variables numéricas por medio de Kolmogórov-Smirnov encontrando una población de libre distribución, por tal motivo se decide utilizar pruebas no paramétricas.

Se analizó por medio de U de Mann-Whitney, comparando las medianas de la población con HIV vs sin HIV en las variables: peso al nacer, 
talla al nacer, semanas de gestación, días de intubación y días en la UCIN. Posteriormente se hizo una análisis bivariado por medio de correlación de Spearman en las variables: días de estancia en UCIN-peso al nacer, días de estancia en UCIN-talla al nacer, días de estancia en UCIN-semanas de gestación, días de intubación-peso al nacer, días de intubación-talla al nacer, días de intubación-edad gestacional.

\section{Resultados}

Se estudió un grupo de 69 neonatos atendidos en el programa de estimulación temprana del hospital del niño y la mujer SESEQ, de los cuales el $65.2 \%$ (45) son mujeres y el $34.3 \%$ (24) varones. Tuvieron una edad gestacional de $34.19 \pm 4.22$ semanas, peso al nacer de $2116.09 \pm 859.36 \mathrm{~g} \mathrm{y}$ talla de $44.06 \pm 6.02 \mathrm{~cm}$. La estancia en UCIN para la muestra fue de 11.88 \pm 16.84 días y estuvieron $8.65 \pm 12.20$ días con ventilación mecánica invasiva. Dentro de los antecedentes de la madre se encontró una edad media de $27.28 \pm 6.54$, número de gestas previas $2.38 \pm 1.7$ y número de partos previos $1.01 \pm 1.52$. Se observaron 25 casos de HIV diagnosticados por medio de ultrasonido transfontanelar de los cuales 7 (10\%) casos son HIV grado I, $14(20 \%)$ II, 4 (5.7\%) III y no se diagnosticaron casos de grado IV.

La Tabla 1 muestra los factores que tiene relación con HIV donde se encontró que: del total de los niños con diagnóstico de HIV 20 (80.0\%) se obtuvieron mediante cesárea, no se registraron casos de distocias. Un total de 5 pacientes $(20.0 \%)$ tienen antecedentes de preclampsia, 1 (4\%) caso requirió de reanimación neonatal con compresiones torácicas.

Del total de la población estudiada $21(84.0 \%)$ fue diagnosticado con hiperbilirrubinemia, $24(96.0 \%)$ sepsis, $5(8.0 \%)$ enterocolitis necrosante, 2 (8.0\%) neumotórax, $2(8.0 \%)$ asfixia, 1 (4.0\%) conducto arterioso sintomático, $2(8.0 \%)$ cardiopatía congénita, 19 (84\%) síndrome de membrana hialina.

Por su parte $23(92 \%)$ de los neonatos ingresaron a la UCIN, 9 (42.9\%) fueron prematuros extremos, 4 (19\%) pesaron menos de $1000 \mathrm{~g}$ al nacer. $11(55 \%)$ pasaron más de una semana con intubación y $17(81.0 \%)$ permanecieron más de una semana en la UCIN.

Tabla 1. Factores del neonato con HIV

\begin{tabular}{lll}
\hline Factor & Frecuencia & $\%$ \\
\hline Sepsis & 24 & 98 \\
Necesidad de intubación & 24 & 98 \\
Hiperbilirrubinemia & 21 & 94 \\
Membrana Hialina & 19 & 84 \\
Peso < 1500 g & 15 & 60 \\
Prematuro extremo & 9 & 36 \\
Apoyo aminérgico & 9 & 36 \\
Enterocolitos necrosante & 5 & 20 \\
Parto transvaginal & 5 & 20
\end{tabular}


Preclampsia de la madre

Neumotórax

Cardiopatía congénita

Asfixia

Conducto arterioso sintomático

Reanimación avanzada

\begin{tabular}{ll}
5 & 20 \\
2 & 8 \\
2 & 8 \\
2 & 8 \\
1 & 4 \\
1 & 4 \\
\hline & HENM 2015
\end{tabular}

Como se puede observar en la Tabla 2 los antecedentes más relevantes en pacientes con presencia de HIV grado III y IV son sepsis 4 $(100 \%)$, síndrome de membrana hialina 4 (100\%), prematuro extremo 3 (75\%), y necesidad de intubación 3 (75\%).

Tabla 2. Factores del neonato con HIV III y IV

\begin{tabular}{lll}
\hline Factores & Frecuencia & $\%$ \\
\hline Sepsis & 4 & 100 \\
Membrana Hialina & 4 & 100 \\
Necesidad de intubación & 3 & 75 \\
Prematuro extremo & 3 & 75 \\
Peso < $1500 \mathrm{~g}$ & 2 & 50 \\
Apoyo aminergico & 2 & 50 \\
Parto transvaginal & 2 & 50 \\
Hiperbilirrubinemia & 1 & 25 \\
Enterocolitos necrosante & 1 & 25 \\
Conducto arterioso sintomático & 1 & 25 \\
\hline $\mathrm{n}=4$ & & HENM 2015
\end{tabular}

Se realizó una comparación entre los niños con HIV y sin HIV. Se hizo una prueba de distribución normal de la muestra por medio de Kolmogórov-Smirnov en la variables semanas de gestación, peso al nacer, talla al nacer, APGAR, días de estancia en la UCIN y días de intubación con un $\mathrm{p}$ valor $<0.05$ en todas ellas, lo cual es sugerente de una muestra de libre distribución y de necesidad de pruebas no paramétricas.

Posteriormente como se muestra en la tabla 3 utilizando la $\mathrm{U}$ de Mann-Whitney para comparación de medianas se encuentra una diferencia significativa, teniendo una $\mathrm{p}<0.05$, entre el grupo de neonatos con HIV y sin HIV en las variables cuantitativas de: semanas de gestación (Figura 1), peso (Figura 2), talla (Figura 3), días de intubación, APGAR y días de estancia en UCIN. Al momento de comparar las medianas entre géneros se observó igualdad en las variables numéricas anteriormente mencionadas. Las variables en las cuales no se encuentra diferencia estadística entre los pacientes con y sin diagnóstico de HIV son: edad de la madre, número de gestas previas y número de partos previos. 
Tabla 3. Comparación de grupo con HIV y sin HIV

\begin{tabular}{|l|ll|ll|l|}
\multicolumn{5}{|c|}{ HIV } \\
\hline Factor de riesgo & Media & \pm & Media & \pm & p valor \\
\hline Semanas de gestación & 30.84 & 2.93 & 36.09 & 3.63 & 0.000 \\
Talla al nacer & 39.48 & 5.16 & 46.66 & 4.84 & 0.000 \\
Peso al nacer & 1468.00 & 507.98 & 2484.32 & 800.55 & 0.000 \\
APGAR & 7.84 & 1.06 & 8.36 & 0.87 & 0.041 \\
Días UCI & 25.84 & 3.95 & 18.30 & 9.10 & 0.000 \\
Días de intubación & 16.16 & 4.07 & 14.88 & 7.24 & 0.000 \\
\hline
\end{tabular}

$\mathrm{n}=69 \quad$ HENM, 2015

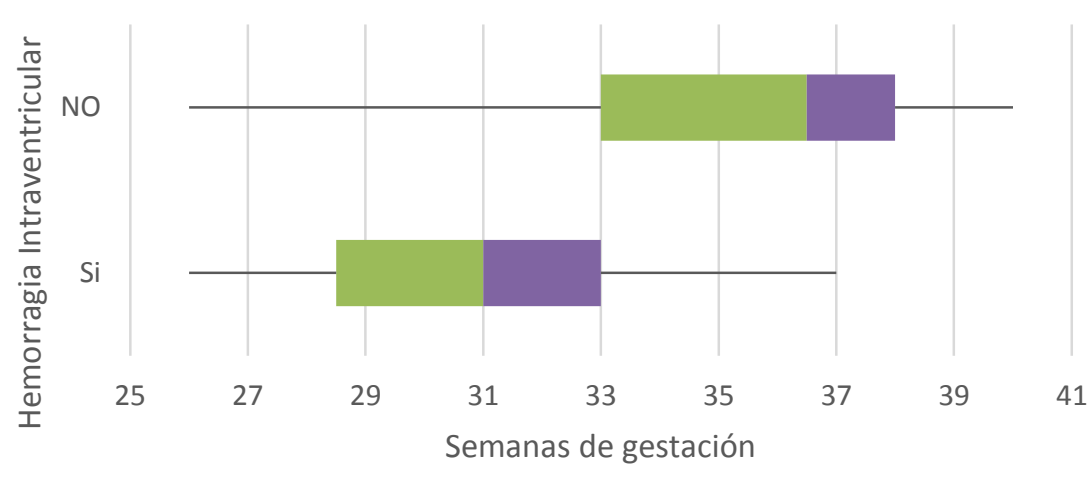

$\mathrm{n}=69$

Figura 1. Semanas de gestación con y sin HIV. HENM 2015

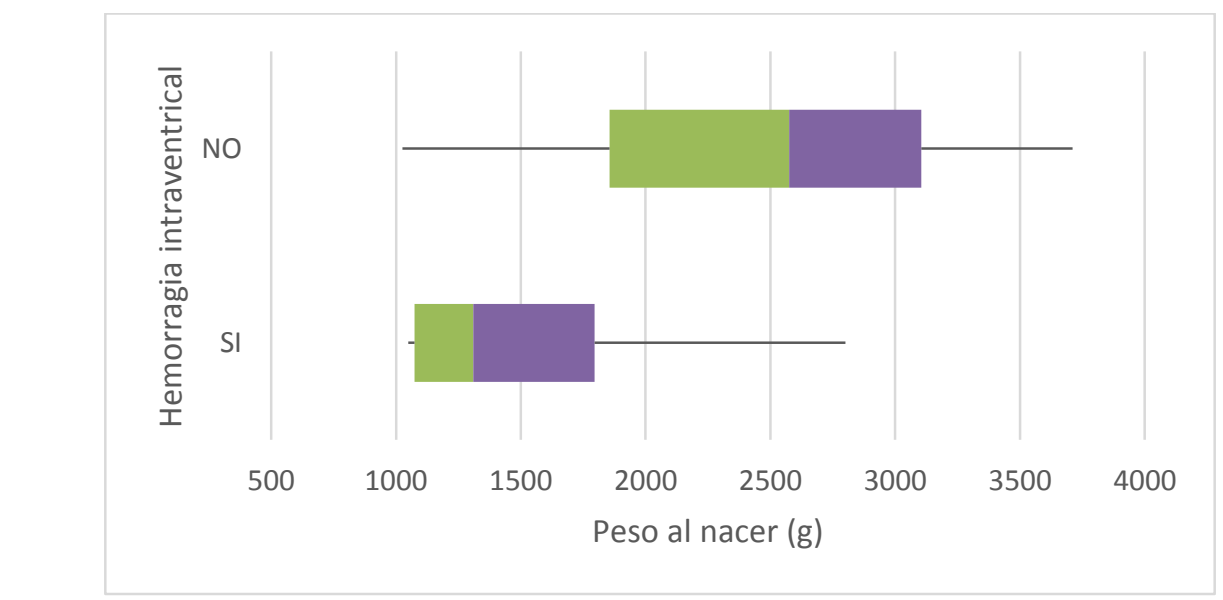

$\mathrm{n}=69$

Figura 2. Peso al hacer con y sin HIV. HENM 2015 


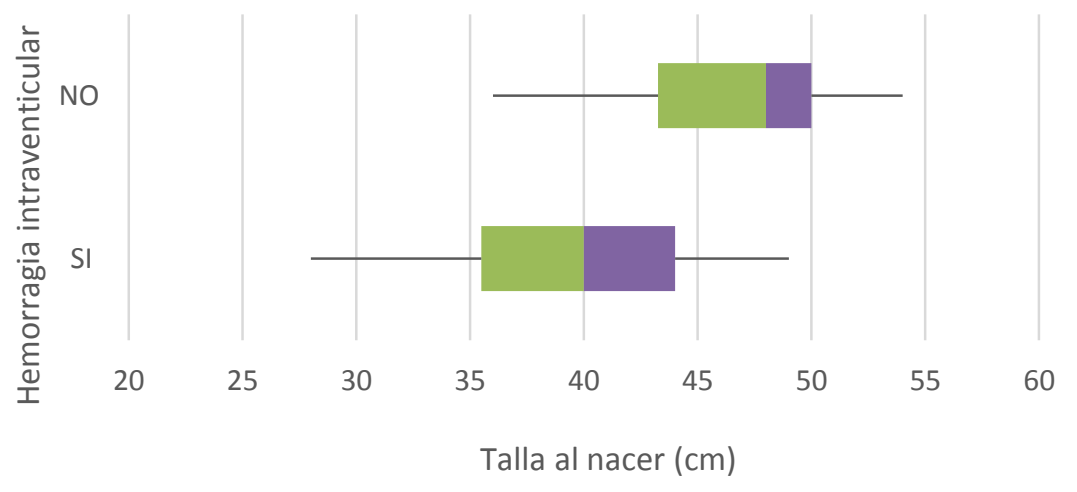

$\mathrm{n}=69$

Figura 3. Talla al nacer con y sin HIV. HENM 2015

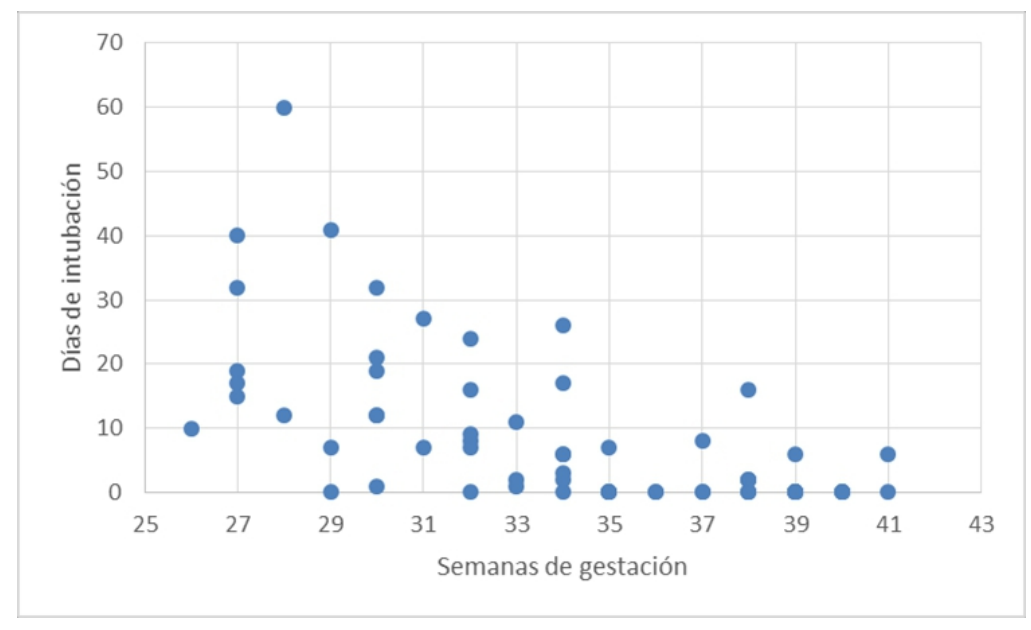

$\mathrm{n}=69$

Fig. 4. Correlación entre días de intubación y semanas de gestación HENM, 2015

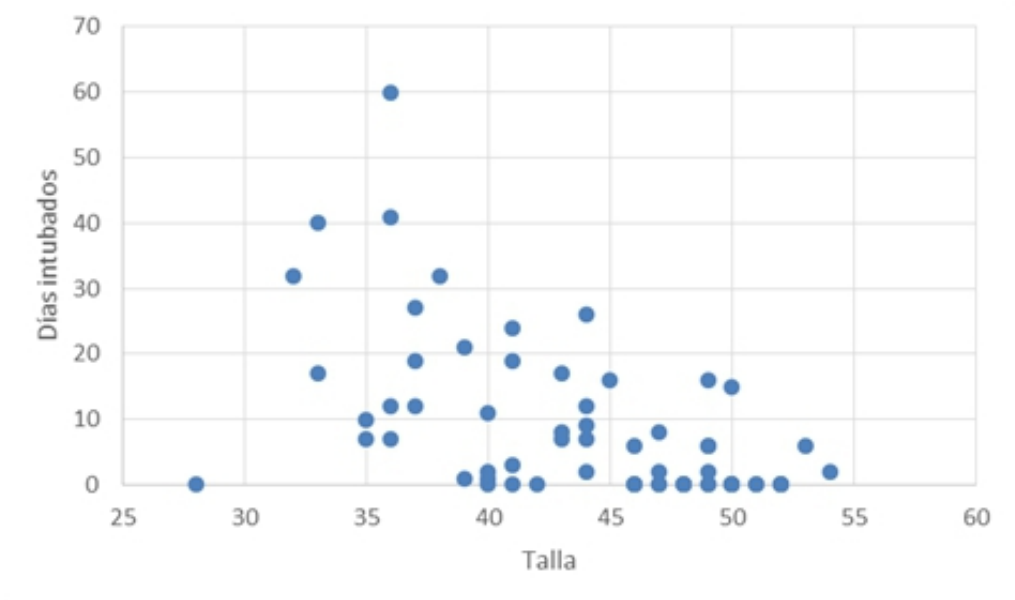

$\mathrm{n}=69$

Figura 5. Correlación entre días de intubación y talla al nacer HENM, 2015 


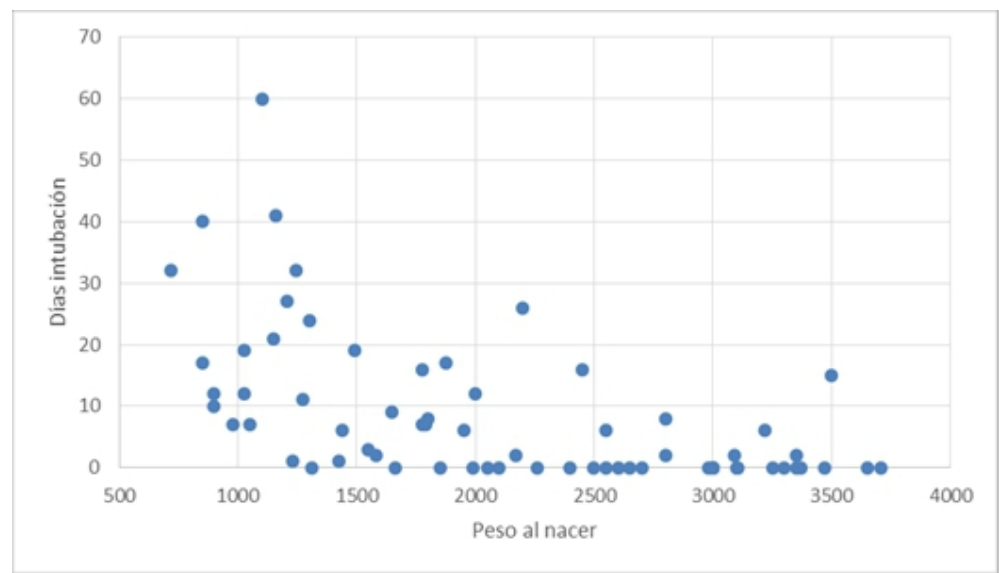

$\mathrm{n}=69$

Figura 6. Correlación entre días de intubación y peso al nacer HENM, 2015

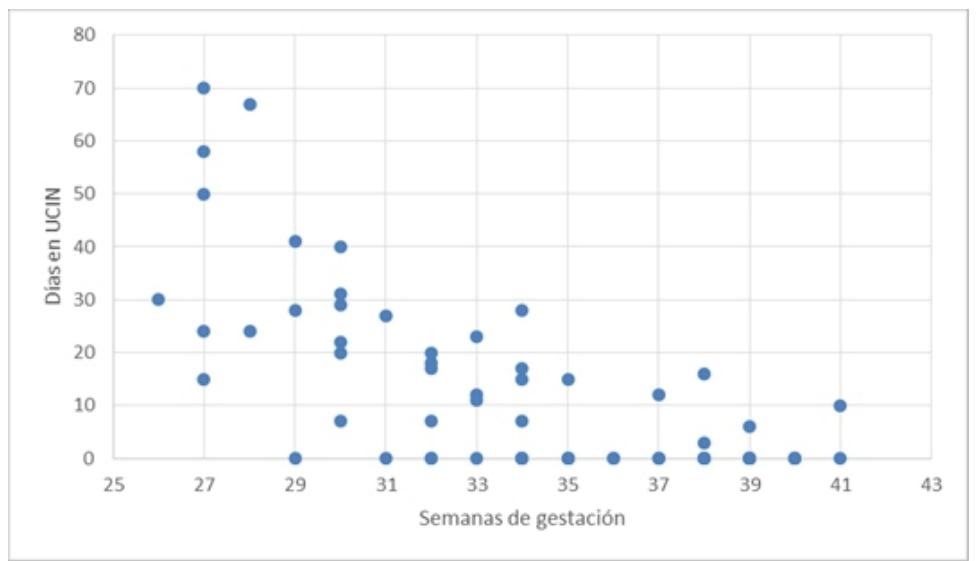

$\mathrm{n}=69$

Figura 7. Correlación entre días de estancia en UCIN y semanas de gestación. HENM, 2015

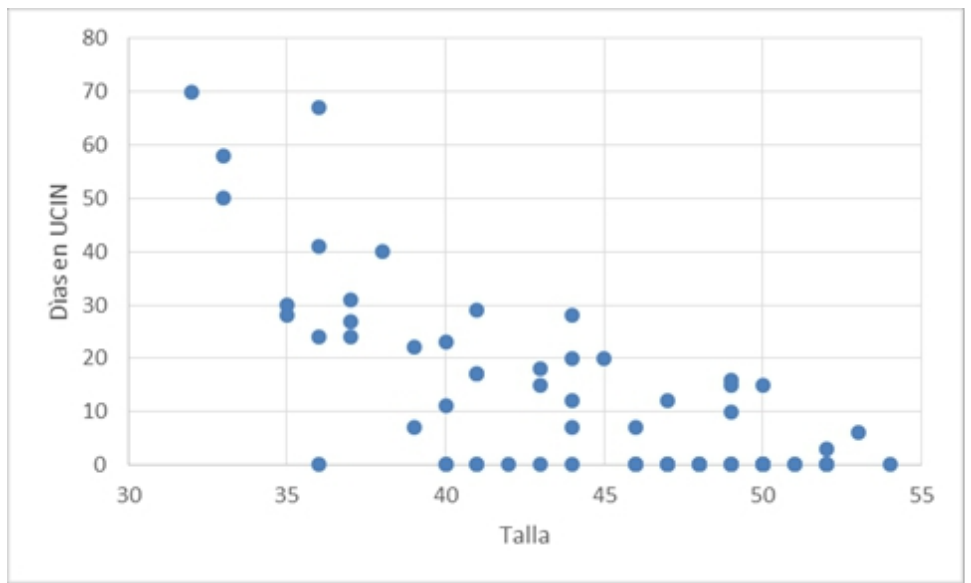

$n=69$

Fig 8. Correlación entre días en UCIN y talla al nacer. HENM, 2015 


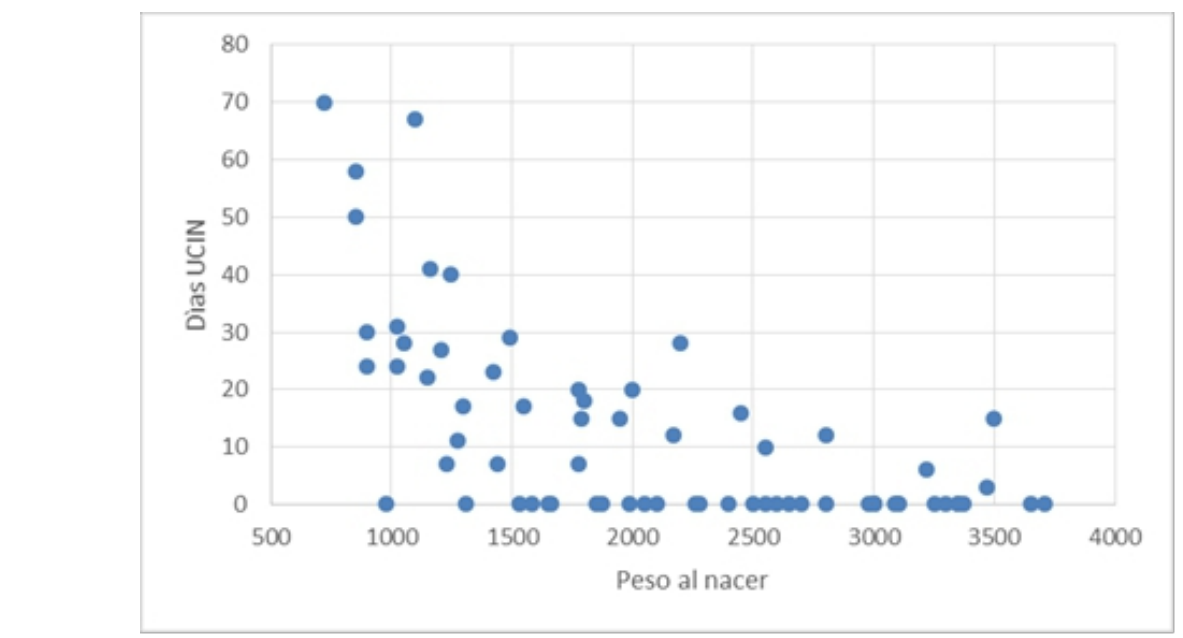

$\mathrm{n}=69$

Figura 9.Correlación entre días en UCIN y peso al nacer HENM, 2015

De manera adicional al estudio se realizó análisis bivariado para observar la relación de los factores estudiados con los días de estandia en UCIN y días de ventilación mecánica por medio de intubación endotraqueal. Por medio de la prueba de Spearman se observó correlación negativa en días de intubación: semanas de gestación (-0.714) (Figura 4), talla (-0.599) (Figura 5) y peso(-0.559) (Figura 6) con un nivel se significancia al nivel de 0.01. En las demás variables numéricas no se encontró correlación significativa A su vez se hizo el mismo análisis para estancia en la UCIN con un nivel de significancia al nivel de 0.01 en las variables: semanas de gestación (-0.708) (Figura 7), talla (- 0.607) (Figura 8), y peso (-0.662) (Figura 9).

Los resultados obtenidos en esta investigación coinciden con los dados a nivel nacional e internacional en donde se ha observado que el antecedente más importante del neonato con hemorragia intraventricular es el bajo peso al nacer y la prematurez. Un estudio de cohorte noruego muestra el seguimiento a 1,764,509 recien nacidos vivos, se observó una mayor incidencia de parálisis cerebral en neonatos prematuos (Tronnes, 2014), si a eso se le suma que el principal tipo de parálisis cerebral es la diplejía espástica a consecuencia de lesión en la sustancia blanca periventricular secundaria a hemorragia intraventricular (Euson, 2012; Maenner, 2016), se vuelve patente la importancia de la HIV en la génesis de discapacidad en paciente pediátrico.

\section{Conclusión}

Debido a que existe diferencia entre los grupos de estudio en las variables de peso, talla, semanas de gestación, días de UCIN y días de 
intubación (neonatos con HIV y sin HIV) se debe continuar con las investigaciones para recopilar una mayor cantidad de información. La labilidad de la matriz germinal subependimaria podría explicar por qué existe una diferencia entre los grupos en días de estancia en UCIN y/o ventilación mecánica invasiva. Los neonatos bajo estas condiciones presentan alteración hemodinámica que pudiera propiciar la aparición de HIV.

Resulta fundamental fortalecer los programas de prevención de los partos prematuros, neuroprotección en la UCIN y aumentar los programas de prevención secundaria sobre todo en cuanto a neurohabilitación se refiere, para minimizar el impacto de la HIV a nivel neurológico y demás alteraciones neurológicas derivadas de la prematurez (Basso, 2016; Euson, 2012). Además se debe mejorar las condiciones generales del recién nacido dentro de la UCIN con un adecuado control de la luz, ruido, temperatura, dolor y respeto los ciclos de sueño vigilia. Es evidente que la protección neurológica del recién nacido es responsabilidad de todo el equipo sanitario de las unidades de neonatología, desde la neuroprotección farmacológica con cafeína, cuidados basados en el desarrollo (Basso, 2016; Torres, 2016), adecuado manejo respiratorio y hemodinámico (Nuñez, 2016), cuidados de enfermería meticulosos para prevenir eventos adversos como hipercapnias o desaturaciones de oxígeno, entro otras cosas. Todo esto, y no solo la implementación de un programa de estimulación-intervención temprana darán como el resultado una reducción en la tasa de discapacidad y mortalidad de neonatos en nuestro país.

\section{References:}

1. Ahn, SY., \& Shim, S. (2015). Intraventricular Hemorrhage and Post Hemorrhagic Hydrocephalus among Very-Low-Birth-Weight Infants in Korea. Journal of Korean Medical Sciencie, 52(8). 52-58.

2. Basso Graciela, MMJ. (2016). Neurodesarrollo en Neonatología. Intervención ultratemprana en la Unidad de Cuidados Intensivos Neonatales. Buenos Aires, Argentina: Medica Panamericana. 221$240 \mathrm{p}$.

3. Christian, EA., Jin, DL., Attenello, F., Wen, T., Cen, S., Mack, WJ. et al. (2016). Trends in hospitalization of preterm infants with intraventricular hemorrhage and hydrocephalus in the United States, 2000-2010. Journal of Neurosurgery: Pediatrics, 17, 260-269.

4. Cervantes-Ruiz, MA., Rivera-Rueda, MA., Yescas-Buendía, GV. et al. (2012). Hemorragia intraventricular en recién nacidos pretérmino en una Unidad de Tercer Nivel en la Ciudad de México. Perinatología y Reproducción Humana, 26(1), 17-24.

5. De Castro, F., Allen-Leigh, B., Katz, G. et al. (2013). Indicadores de bienestar y desarrollo infantil en México. Salud Pública de México, 
55(2), 267-275.

6. Euson, P. (2012). Aetiology and epidemiology of cerebral palsy. Pediatrics and Child Health. 22 (9), 361-366.

7. Ferriero, DM. (2016). The Vulnerable Newborn Brain: Imaging Patterns of Acquired Perinatal Injury. Neonatology, 109 (1), 345-51.

8. Glauco Valdivieso, JCR. (2015). Factores asociados a Hemorragia Intraventricular en Neonatos Prematuros en el Hospital Regional Docente de Trujillo . Diciembre 2011 a Diciembre 2013 . Horizonte médico, 15(2).

9. Liebowitz, M., \& Clyman, RI. (2016). Antenatal Betamethasone: A Prolonged Time Interval from Administration to Delivery Is Associated with an Increased Incidence of Severe Intraventricular Hemorrhage in Infants Born before 28 Weeks Gestation. Journal of Pediatrics, 1(8).

10. Maenner, MJ. et al. (2016).Prevalence of cerebral palsy and intellectual disability among children identified in two U.S. national surveys, 2011-2013. Annals of Epidemiology. 26 (3), 222-226.

11. Moreno-flores, RLA. (2016). El ABC de la ecografía transfontanelar y más. Radiologia, 2(7).

12. Mukerji, A., Shah, V., Shah, PS. (2015). Periventricular / Intraventricular Hemorrhage and Neurodevelopmental Outcomes : A Meta-analysis. Journal of Pediatrics, 136(6).

13. Nuñez, JR., \& Orozco, A. (2016). Estabilización temprana del recién nacido pretérmino menor de 1,200 gramos. Acta Médica del Grupo Ángeles, 14(1), 25-31.

14. Rivera, F. \& Tori, A. (2014). Incidencia de la hemorragia intraventricular en prematuros de muy bajo peso y sus factores asociados en un hospital nacional de Lima, Perú. Revista Médica Herediana, 25, 60-67.

15. Torres, J., Palencia, D., Sanchéz, DM. et al. (2016). Programa Madre Canguro: primeros resultados de una cohorte de niños seguidos desde la unidad neonatal hasta la semana 40 de edad postconcepcional. Colombia Médica, 37 (2) 96-101.

16. Tronnes H, WIlcox AJ et al. (2014). Risk of Cerebral Palsy in relation to pregnacy disorders and preterm birth: a national cohort study.Developmental Medicine \& Child Neurology, 56(8): 779-785.

17. Vesoulis, ZA., Ters N El, Foster, A., Trivedi, SB., Liao, SM. \& Mathur, AM. (2016). Response to dopamine in prematurity: a biomarker for brain injury? Journal of Perinatology, 36(6):453-8.

18. Wusthoff, CJ. (2016). Intracranial Hemorrhage in the Neonate. Neonatal Network ,35(2),67-71. 